\title{
Time domain oscillating poles: Stability redefined in Memristor based Wien-oscillator
}

\author{
A. Talukdar ${ }^{1}$, A. G. Radwan ${ }^{1,2}$, and K. N. Salama ${ }^{1}$ \\ ${ }^{1}$ Electrical Engineering Program \\ King Abdullah University of Science and Technology (KAUST) \\ Thuwal, Kingdom of Saudi Arabia 23955-6900 \\ ${ }^{2}$ Department of Engineering Mathematics \\ Faculty of Engineering, Cairo University, Egypt \\ Email: \{abdul.talukdar, ahmed.radwan, khaled.salama\}@kaust.edu.sa
}

\begin{abstract}
Traditionally, the necessary and sufficient condition for any system to be oscillating is that its poles are located on the imaginary $(j \omega)$ axis. In this paper, for the first time, we have shown that systems can oscillate with time-domain oscillating poles. The idea is verified using a Memristor based Wien oscillator. Sustained oscillations are observed without having the poles of the system fixed on the imaginary axis and the oscillating behavior of the system poles is reported. The oscillating resistance and triangular shape of FFT are also demonstrated with mathematical reasoning and simulation results to support the unusual and surprising characteristics.
\end{abstract}

Index Terms-Memristor, oscillating poles, stable oscillation

\section{INTRODUCTION}

In 1971 Leon O. Chua $[1,2]$ showed the existence of a new two-terminal circuit element called the 'Memristor'. He proposed the Memristor as the fourth basic circuit element which relates between the flux-linkage $(\varphi)$ and the charge (q). The unconventional properties of Memristor have led to the successful modeling of a number of physical devices and systems [3-5]. Interest in the Memristor revived in 2008 when an experimental version was reported by HP lab [6]. That device neither uses magnetic flux, nor stores charge as a capacitor does, rather achieves a resistance dependent on the history of current. The Memristor has been investigated independently or with the other three passive components $(\mathrm{R}$, $\mathrm{L}$, and C) $[7,8]$. Some patents related to Memristors appear to include applications in DRAM [9], programmable logic [9], signal processing [10], neural networks [11], and control systems [12].

Oscillators generally use some form of active device: opamp or BJT, surrounded by passive devices such as resistors, capacitors, and inductors, to generate a sinusoidal output [13]. They are widely used in generating signals to broadcast by radio and television transmitters, clock signals that regulate computers and quartz clocks. The Wien oscillator is one of the simplest and best known oscillators.

Poles of an oscillatory system play the major role in determining the stability of oscillation [13]. The location of the poles and the values of the real and imaginary parts determine the response of the system. The condition for any system to oscillate is achieved by forcing the poles to have only imaginary part and these poles should have a fixed position.

In this article we will describe a new phenomenon caused by employing a Memristor in a conventional Wien oscillator circuit. A mathematical model is proposed to analyze Wien Memristor-oscillator. The simulation results and mathematical models presented here demonstrate the surprising findings in the field of oscillation which represent a new paradigm in circuit theory.

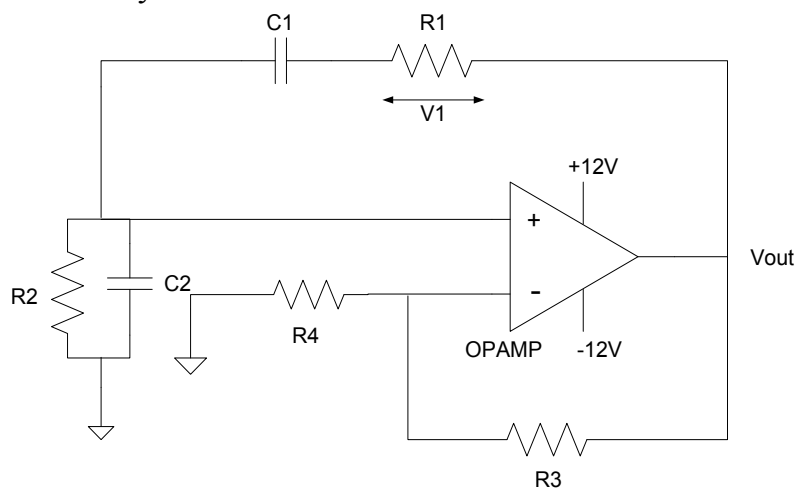

Fig. 1: Schematics of conventional Wien oscillator

\section{OVERVIEW OF WIEN OSCILLATOR}

\section{A. Wien Oscillator}

The Wien bridge oscillator is one of the various types of electronic oscillators that can generate sine waves. It can generate a large range of frequencies. The main components of this oscillator are four resistors and two capacitors [13]. Opamp sine-wave oscillators operate without an externallyapplied input signal. Some combinations of positive and negative feedback are used to drive the op amp into an unstable state and this causes the output to oscillate. The derived equations for the frequency of oscillation and the condition of oscillation are demonstrated later. The schematic of the basic Wien oscillator is shown in Fig. 1. 


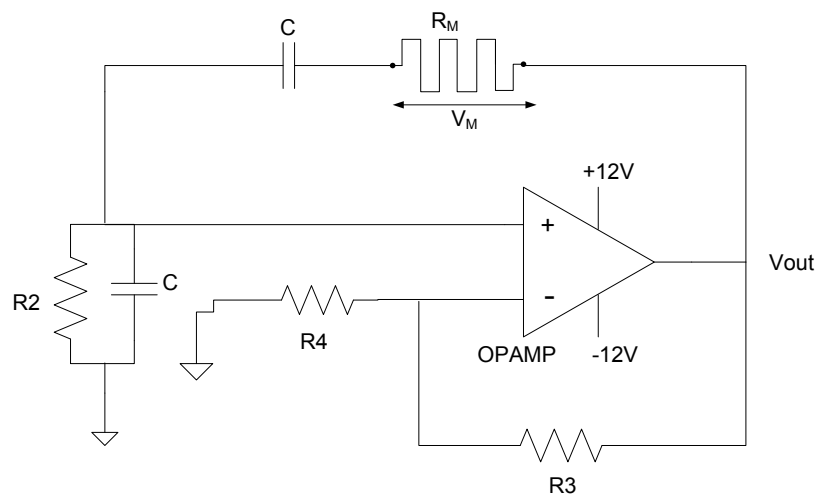

Fig. 2: Schematics of Wien oscillator with Memristor

Considering the circuit shown in Fig. 1 the characteristics equation of this system can be derived in the following form:

$$
\begin{gathered}
s^{2}+b s+d=0 \\
b=\frac{1}{R_{2} C_{2}}+\frac{1}{R_{1} C_{1}}-\frac{R_{3}}{R_{1} R_{4} C_{2}}, d=\frac{1}{R_{1} R_{2} C_{1} C_{2}}
\end{gathered}
$$

The condition of sustained oscillation is given by

$$
\frac{C_{2}}{C_{1}}+\frac{R_{1}}{R_{2}}=\frac{R_{3}}{R_{4}}
$$

The frequency of oscillation is expressed as:

$$
f=\frac{1}{2 \pi \sqrt{R_{1} R_{2} C_{1} C_{2}}}
$$

For sustain oscillation $b$ must be equal to zero in (1). The location of the poles in this system can be found by solving the quadratic equation in (1). When $b=0$, there will be two conjugate poles lying on the imaginary axis. If $b$ is a non zero value then those two poles will be located in either the right half or left half of the s-plane. For oscillations to be sustained, the poles of the system must lie on the imaginary axis. . Shifting of poles from the imaginary axis will result in a damped oscillation, which is not a sustained oscillation.

\section{B. Wien Oscillator with Memristor}

Referred to Fig. 1, $\mathrm{R}_{1}$ is replaced with Memristor and both $\mathrm{C}_{1}$ and $\mathrm{C}_{2}$ are changed to same valued capacitance $\mathrm{C}$ as shown in Fig. 2. The resistance of the Memristor will be designated as $R_{M}$ and the voltage across the Memristor will be designated as $\mathrm{V}_{\mathrm{M}}$. From the simulation results $\mathrm{R}_{\mathrm{M}}$ is found to be oscillating. So $R_{M}$ will have an average value which can be denoted as $\mathrm{R}_{\text {avg. }}$. These changes will certainly modify equations (1)-(4). The frequency of oscillation of the new system, $\mathrm{f}_{\mathrm{M}}$ can be written as:

$$
f_{M}=\frac{1}{2 \pi C \sqrt{R_{\text {avg }} R_{2}}}
$$

The condition of oscillation is modified as:

$$
1+\frac{R_{\text {avg }}}{R_{2}}=\frac{R_{3}}{R_{4}} \equiv \text { gain }
$$

Dmitri B. Strukov et al. first proposed a physical model to realize the Memristor as a two terminal device [6]. They considered a thin semiconductor film of thickness sandwiched between two metal contacts. The semiconductor film has a region with a high concentration of dopants having

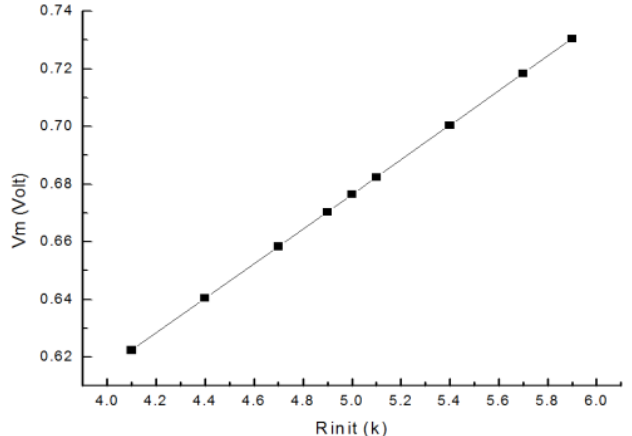

Fig. 3: Voltage across the Memristor $\left(V_{M}\right)$ from simulation

low resistance $R_{\text {on }}$, and the remainder has a low dopant concentration and higher resistance $\mathrm{R}_{\text {off }}$. The total resistance of the Memristor, $\mathrm{R}_{\mathrm{M}}$, is a sum of the weighted (depending on the width of the doped and undoped regions) resistances of the doped and undoped regions. The SPICE model proposed by Zdeněk Biolek et al. $[14,15]$ can be used to simulate the effect of Memristor in various circuits. The model is implemented as a SPICE subcircuit with the following parameters: the initial $R_{\text {init }}$ resistance, the $R_{\text {off }}$ and $R_{\text {on }}$ resistances, the width of the thin film $D$, the dopant mobility $\mu_{v}$, and the exponent $p$ of the window function. To counterpart the effect of the nonlinear dopant drift they have proposed a window function with $p$ as the parameter to set the nonlinearity. The differences between models with linear and nonlinear drifts decrease if $p$ increases. Recent mathematical modeling of Memristor in case of periodic signals is proposed in $[16,17]$.

For our simulations we have used $\mathrm{R}_{2}=5 \mathrm{k}, \mathrm{C}_{1}=\mathrm{C}_{2}=\mathrm{C}=3.2 \mu \mathrm{F}$, $\mathrm{R}_{4}=100 \mathrm{k}, \mathrm{R}_{1}$ (in Fig. 1) is replaced with Memristor (Fig. 2). In the SPICE model only $\mathrm{R}_{\text {init }}$ is changed and the value of $p$ is given 10. $p=10$ is good enough for linear approximation. The other parameters were kept as it is in the model file [15]. $R_{\text {init }}$ was changed from $4.1 \mathrm{k}$ to $5.9 \mathrm{k}$ for simulation and also for mathematical modeling.

\section{RESULTS AND DISCUSSION}

Sustained oscillations are found for each $R_{\text {init }}$ value of $R_{M}$. In Fig. 3 the simulated values of $V_{M}$ (voltage across the Memristor) is shown for every value of $\mathrm{R}_{\text {init. }}$. From this figure it is easily observed that $V_{M}$ has linear relation with $R_{\text {init. }}$. So $\mathrm{V}_{\mathrm{M}}$ (the peak voltage of $\mathrm{V}_{\mathrm{M}}$ ) can be modeled with straight line approximation. $\mathrm{V}_{\mathrm{M}}$ can be expressed as a function of $\mathrm{R}_{\text {init }}$ :

$$
V_{M}=0.3764+0.06 R_{\text {init }}
$$

The calculated values of $\mathrm{V}_{\mathrm{M}}$ using (7) give similar results as simulation and the error in this case is less than $0.22 \%$. Thus $\mathrm{V}_{\mathrm{M}}$ can be approximated by two methods: the first method is by using (7) where $V_{M}$ can be calculated for every $R_{\text {init. }}$. The second method would be to use conventional Wien oscillator (Fig. 1). As the average values of $\mathrm{V}_{\mathrm{M}}$ are almost constant whether a Memristor is used or not if similar values of $R_{1}$ and $R_{\text {init }}$ are used so $V_{1}$ can be taken from conventional Wien oscillator to be used as $V_{M}$ in the proposed formula described later. In Fig. 4 the simulation result is shown for $R_{M}$ and $V_{\text {out }}$. $V_{\text {out }}$ is the final output voltage. For this simulation, $R_{\text {init }}=5 \mathrm{k}$. Similar results are found for other values of $R_{\text {init. }}$ The sustained oscillation is clearly observed in both the final output $V_{\text {out }}$ andR $R_{M}$ (Fig. 4). $R_{3}$ has to be changed to 
accomplish this oscillation. The values of $\mathrm{R}_{3}$ are found equal to the calculated values derived from (6). In this case $R_{\text {init }}$ is taken as $R_{\text {avg. }}$. This approximation does not alter the result as $R_{\text {avg }}$ (from the simulation) is very close to $R_{\text {init }}$ with an error less than $0.02 \%$. The most interesting observation is that the output oscillation is sustained as shown in Fig. 4. Though from the SPICE model [15] $R_{M}$ can take values like $R_{o n}=0.1 k$, $R_{\text {off }}=16 \mathrm{k}$, and $R_{\text {init }}$ but from the simulation it is found that $R_{M}$ is oscillating in a different range across $\mathrm{R}_{\text {avg. }}$. So Memristor resistance, $R_{M}$ can be modeled as:

$$
R_{M}=R_{\text {avg }}+\Delta R_{M} \sin (\omega t \pm \varphi)
$$

Where, $\Delta R_{M}$ is the sinusoidal amplitude. If only the magnitude peaks of $\mathrm{R}_{\mathrm{M}}$ is considered then (8) can be written as:

$$
R_{\text {max } \min }=R_{\text {avg }} \pm \Delta R_{M}
$$

As $R_{M}$ is oscillating, It will have a maximum $\left(R_{\max }\right)$ and a minimum $\left(\mathrm{R}_{\min }\right)$ values. Using the concept described in [16] and reusing (5) and (6) the maximum and minimum values of $\mathrm{R}_{\mathrm{M}}$ can be modeled as:

$$
R_{\text {max }}^{2}=R_{\text {min }}^{2} \pm\left[\frac{2 V_{M} k\left(R_{\text {off }}-R_{\text {on }}\right)}{\pi f_{M}}\right]
$$

Where, $\mathrm{R}_{\text {on }}$ and $\mathrm{R}_{\text {off }}$ are described previously. $k$ is the same factor as described in [3] and it is given as $k=\left(\mu_{v} R_{o n}\right) / D^{2}$
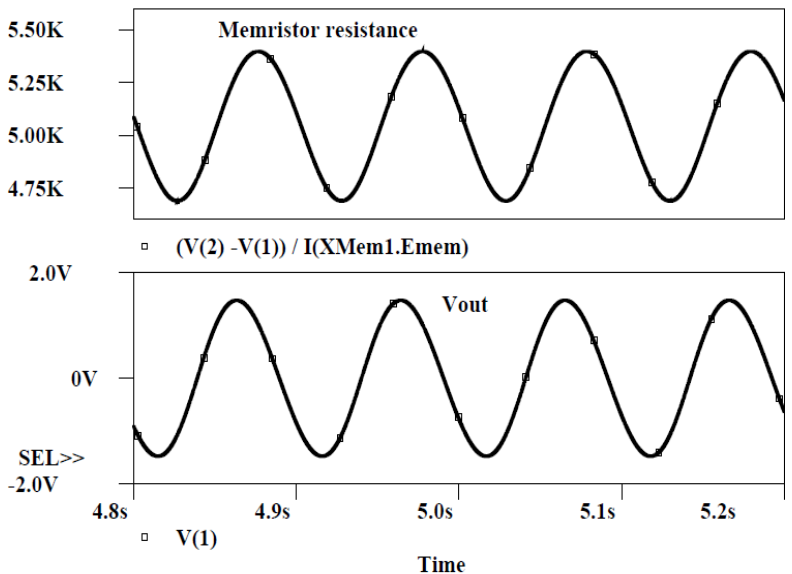

Fig. 4: Simulation result for $R_{M}$ and $V_{\text {out }}$ of the final output oscillation.

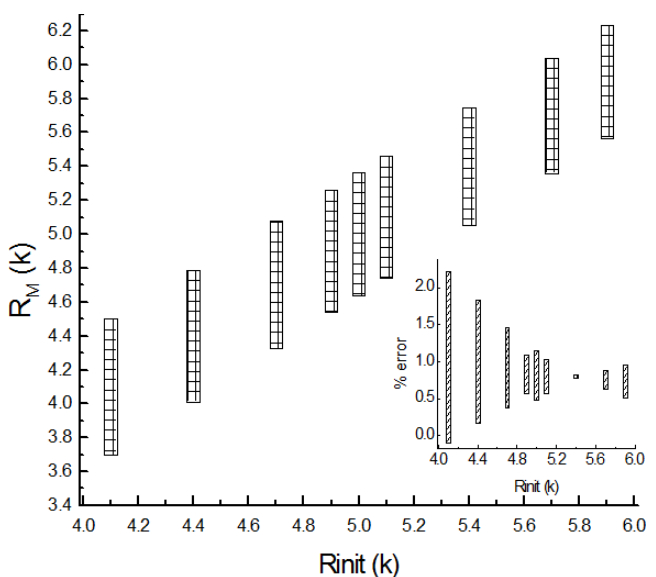

Fig. 5: Range of calculated values of $\mathrm{R}_{\mathrm{M}}$; Range of percentage errors between simulated and calculated $\mathrm{R}_{\mathrm{M}}$ (inset).
Using the bionomial expansion, equation (10) can be approximated as following:

$\left|R_{\text {max }}-R_{\text {init }}\right| \cong\left[\frac{\left\{V_{M} k\left(R_{\text {off }}-R_{\text {on }}\right)\right\}}{\left\{2 \pi R_{\text {init }} f_{\text {avg }}\right\}}\right] \equiv \Delta R_{M}$

For any value of $R_{\text {init }}$, the peaks $R_{\max }$ and $R_{\min }$ can be easily calculated using (11). In Fig. 5 the calculated range of the Memristor resistance $R_{M}$ using (11) is shown for different values of $R_{\text {init. }}$. The inset figure in Fig. 5 shows the percentage error between simulation and calculated values for every range of $\mathrm{R}_{\mathrm{M}}$. From Fig. 5 it is seen that the calculated and the simulated values of $R_{M}$ are quite close with a maximum error of $2.2 \%$ which is negligible.

\section{A. Poles as a function of time: "Oscillating poles"}

It is well known that for any oscillation to be stable, the poles of that system must lie on imaginary axis. In other words, there should not be any real part in the value of the pole. If the poles of the system fall in right half of the $s$ plane, then the system will have a over damped oscillation, which will be increasing by time, and as a result, the oscillation will saturate. If the poles of the system fall in the left half of $s$ plane, the system will have a damped oscillation, which dies out eventually. Using (2), and (6) the expression for $b$ and $d$ can be written as:

$$
b=\frac{1}{C R_{M} R_{2}}\left[R_{M}-R_{\text {avg }}\right] \cong \frac{\Delta R_{M}}{C R_{M} R_{2}}, d=\frac{1}{C^{2} R_{M} R_{2}}
$$

The solution for $s$ can be written as:

$$
s=\sigma \pm j \omega=\frac{b}{2} \pm j\left[\frac{\sqrt{4 R_{M} R_{2}-\left(\Delta R_{M}\right)^{2}}}{2 C R_{M} R_{2}}\right]
$$

Conventional stable oscillation can only be achieved if $b=0$ as in case of the Wien oscillator presented in Fig. 1 where the oscillation is sustained by adjusting the values of $R_{1}, R_{2}, C_{1}$, $\mathrm{C}_{2}, \mathrm{R}_{3}$, and $\mathrm{R}_{4}$. But in the case where a Memristor is used (refer to (12)), $b$ is found directly proportional to $\Delta \mathrm{R}_{\mathrm{M}}$. So a small value of $\Delta \mathrm{R}_{\mathrm{M}}$ can give a significant value of $b$. If $\Delta R_{M}=0$, poles will be on $\pm j \omega$ axis. These poles can be named as "fixed poles". In Fig. 4 we can see the resistance of Memristor, $\mathrm{R}_{\mathrm{M}}$ is oscillating and so definitely $\Delta \mathrm{R}_{\mathrm{M}} \neq 0$ and so $b$ cannot be zero. As a result the poles will not be fixed. But still sustained oscillation is found and it is clear in the simulation result of $V_{\text {out }}$ (in Fig. 4). These poles can be named as "oscillating poles". In Fig. 6, the oscillating nature of poles is shown. In Fig. 6(a), $R_{M}$ is plotted using (8) and (11). This result is almost similar to the simulation result of $R_{M}$ shown in Fig. 4. As $R_{M}$ is oscillating so $R_{M}$ can be replaced using (8) in (12) to plot the real part of pole in (13). The plot is shown for $\mathrm{R}_{\text {init }}=5 \mathrm{k}$ and $\mathrm{R}_{\mathrm{avg}}=5.045 \mathrm{k}$. In the same way the imaginary part of pole is plotted in Fig. 6(c). For both cases the oscillating behavior of pole is found. Fig. 6(d) shows the complex pole of the system when $R_{M}$ is changing from its minimum to maximum value. In this figure $+\mathrm{j} \omega$ axis is shown only. The mirror image is found in $-j \omega$ axis. When $R_{M}$ is at its maximum value the poleswill be at the lowest point of the straight line (in Fig. 6(d)). When $R_{M}$ is at its minimum value the poles will be at the highest point of the line (in Fig. 6(d)). The pole will oscillate between these two points along the line for the whole period of oscillation. The pole for which this 
line crosses $+j \omega$ axis corresponds to $R_{a v g}$. Thus the poles of this system will continue to oscillate with time.

\section{B. Frequency response}

As $R_{M}$ is oscillating, the frequency of oscillation $f_{M}$ can be calculated by replacing $\mathrm{R}_{\mathrm{M}}$ with $R_{\text {avg }} \pm \Delta R_{M}$ in (13). It is found that the frequency of oscillation will have a maximum and a minimum value. As the poles of this system are oscillating so the oscillation cannot have a single frequency component in Fourier spectra like in the case of the conventional Wien oscillator. This logical interpretation is well supported by the simulation result shown in Fig. 7. Here the Fourier spectrum is shown for the same transient response of $\mathrm{V}_{\text {out }}$ shown in Fig. 4. The center frequency, $\mathrm{f}_{\mathrm{M} \text {,avg }}=9.90 \mathrm{~Hz}$ corresponds to the purely imaginary pole and the corresponding value of $R_{M}$ is $R_{\text {init. }}$ The calculated values of the maximum and minimum value of $f_{M}$ are found $9.98 \mathrm{~Hz}$ and $9.78 \mathrm{~Hz}$ correspondingly and these values have very close match with $f_{M \text {, max }}$ and $f_{M \text {,min }}$ in Fig. 7 . Other frequencies are due to the shifting of poles from the imaginary axis. This triangular shape of FFT validates the range of frequency of oscillation. It is to be mentioned that for any sustained oscillation the FFT of the sinusoidal oscillation is an impulse. But surprisingly when Memristor is used with Wien oscillator the FFT changes to triangular shape which means that the frequency of oscillation will not be single valued rather it will have a range.

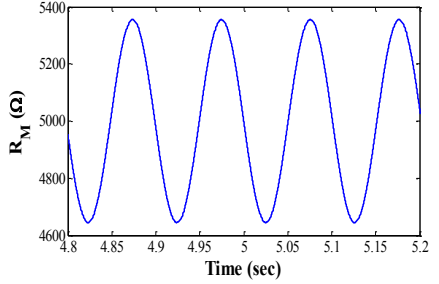

(a)

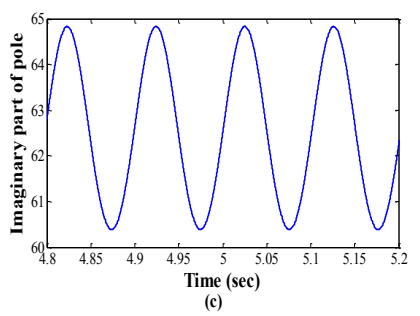

Fig. 6: (a) Oscillating resistance $\mathrm{R}_{\mathrm{M}}$, (b) Oscillating real part of pole, (c) Oscillating imaginary part of pole, and (d) Pole of the described system when $\mathrm{R}_{\mathrm{M}}$ is changing from its minimum to maximum value.

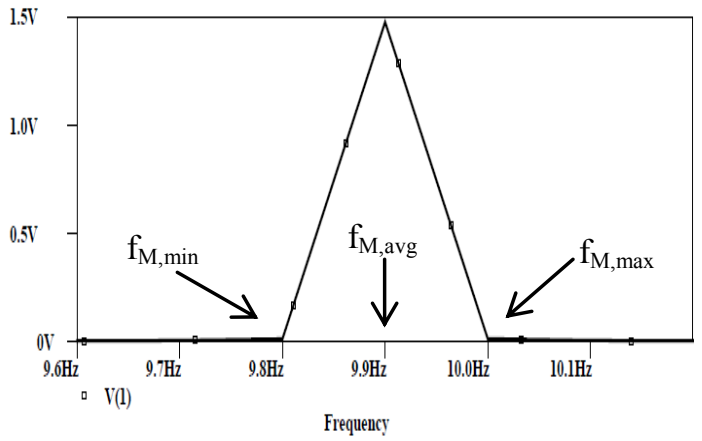

Fig. 7: FFT of output voltage (V(1) in Fig. 4)

\section{CONCLUSION}

In this paper, we reported the effect of using a Memristor in the conventional Wien oscillator. The developed mathematical models for characterizing the oscillation in this scheme match very closely with the simulation results. The new exciting results in oscillation will definitely redefine the traditional concepts. First of all the oscillating behavior of resistance and pole of the investigated scheme can surely give sustained oscillation. Despite of oscillating pole, the stability of the system is not at all diminished. The oscillating resistance, periodic oscillation of poles and triangular shape of FFT may have interesting effect in the field of oscillation. Challenges remain with the nonlinearity of opamp and Memristor itself. The nonlinear characteristics inhibit the complexity of the analysis. The unprecedented results can have impact in designing oscillatory circuits, sensitive control systems, signal generation, amplitude and frequency modulation, etc.

\section{REFERENCES}

[1] L. 0. Chua, "Memristor-The Missing Circuit Element," IEEE Trans. Circuit Theory, vol. CT-18, no. 5, pp. 507-519, Sep. 1971.

[2] L. 0. Chua and S. M. Kang, "Memristive Devices and Systems," Proc.of the IEEE, vol. 64, no. 2, pp. 209-223, Feb. 1976.

[3] G. F. Oster and D. M. Auslander, "The Memristor: A new bondgraph element," Trans ASME on Dynamical Syst. Meas Contr., vol. 94, no. 3, pp. 249-252, 1972.

[4] G. F. Oster, "A note on memristor," IEEE Trans. Circuits Syst., vol. CAS21, pp. 152, Jan. 1974.

[5] L. 0. Chua and C. W. Tseng, "A rnemristive circuit model for P-N junction diodes," Int. J. Circuit Theory and Appl., vol. 2, pp. 367-389, Dec. 1974.

[6] D. B. Strukov, G. S. Snider, D. R. Stuwart, and R. S. Williams, "The missing memristor found," NATURE, vol. 453, pp. 80-83, May 1, 2008.

[7] Di Ventra M., Pershin Y.V., and Chua L.O., "Circuit elements with memory: memristors,memcapacitors and meminductors", Proceedings of IEEE, vol. 97, iss. 10, pp. 1717-1724, Oct 2009.

[8] Qin Yu, Zhiguang Qin, Juebang Yu, and Yuming Mao, "Transmission Characteristics Study of Memristors Based Op-Amp Circuits", ICCCAS 2009, pp. $974-977,2009$.

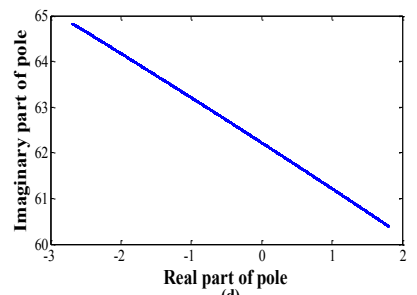

[9] G. Snider, "Architecture and methods for computing with reconfigurable resistor crossbars (Patent style)", U. S. Patent 7203789, April 2007.

[10] B. L. Mouttet, "Programmable crossbar signal processor (Patent style)", U.S. Patent 7302513, Nov 2007.

[11] G. Snider, "Molecular-junction-nanowire-crossbar-based neural network (Patent style)", U.S. Patent 7359888, April 2008

[12] B. L. Mouttet, "Crossbar control circuit (Patent style)", U.S. Patent 7609086, Oct. 2009.

[13] R. Manchini and R. Palmer, "Sine-Wave Oscillators (Report style)", Application Report, SLOA060, Texas Instruments, March 2001

[14] Biolek D., Biolek Z., and Biolkova V., "SPICE Modeling of Memristive, Memcapacitative and Meminductive Systems", European Conference on Circuit Theory and Design (ECCTD), pp. 249-252, 2009.

[15] Z. Biolek, D. Boilek, V. Biolkova, "SPICE Model of Memristor with Nonlinear Dopant Drift," RADIOENGINEERING, vol. 18, no. 2, pp. 210, June 2009.

[16] A. G. Radwan, M. A. Zidan, K. N. Salama, "HP Memristor Mathematical Model for Periodic Signals and DC," IEEE International Midwest Symposium on Circuits and Systems (MWSCAS), pp 861-864, Seattle, USA, Aug 2010.

[17] A. G. Radwan, M. A. Zidan, and K. N. Salama, "On the mathematical modeling of Memristors," IEEE International Conference on Microelectronics, (ICM), Cairo, Egypt, Dec. 2010 